\title{
Outcome of patients on heart transplant list treated with a continuous-flow left ventricular assist device: Insights from the TRans-Atlantic registry on VAd and TrAnsplant (TRAViATA)
} \author{
Antonio Loforte $^{\mathrm{m}}$, Nina Jakus ${ }^{\mathrm{j}}$, Davor Milicic ${ }^{\mathrm{j}}$, Johan Nilsson ${ }^{\mathrm{c}}$, Dina De Bock ${ }^{\mathrm{h}}$, Caroline Sterken ${ }^{\mathrm{i}}$,

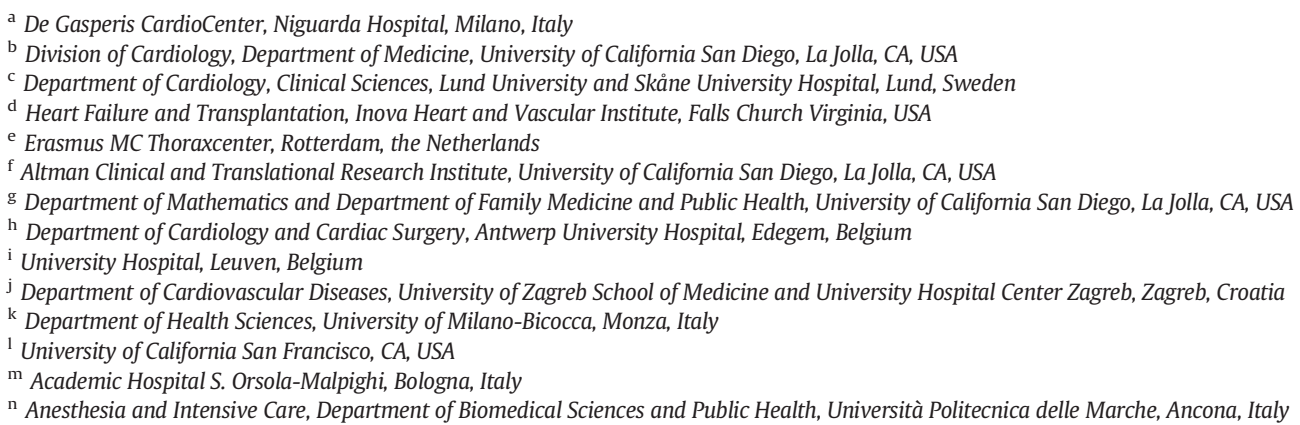

Enrico Ammirati a,*,1, Michela Brambatti ${ }^{\mathrm{b}, 1}$, Oscar Ö. Braun ${ }^{\mathrm{c}}$, Palak Shah ${ }^{\mathrm{d}}$, Manlio Cipriani ${ }^{\mathrm{a}}$, Quan M. Bui ${ }^{\mathrm{b}}$, Jesse Veenis ${ }^{\mathrm{e}}$, Euyhyun Lee ${ }^{\mathrm{f}}$, Ronghui Xu ${ }^{\mathrm{f}, \mathrm{g}}$, Kimberly N. Hong ${ }^{\mathrm{b}}$, Caroline M. Van de Heyning ${ }^{\mathrm{h}}$, Enrico Perna ${ }^{\mathrm{a}}$, Philippe Timmermans ${ }^{i}$, Maja Cikes ${ }^{j}$, Jasper J. Brugts ${ }^{\mathrm{e}}$, Giacomo Veronese ${ }^{\mathrm{a}, \mathrm{k}}$, Jonathan Minto ${ }^{\mathrm{d}}$, Saige Smith ${ }^{\mathrm{b}}$, Grunde Gjesdal $^{\mathrm{c}}$, Yan K. Gernhofer ${ }^{\mathrm{b}}$, Cynthia Partida ${ }^{1}$, Luciano Potena ${ }^{\mathrm{m}}$, Marco Masetti ${ }^{\mathrm{m}}$, Silvia Boschi ${ }^{\mathrm{m}}$, Klaartje Van den Bossche ${ }^{\mathrm{i}}$, Filip Rega ${ }^{\mathrm{i}}$, Hao Tran ${ }^{\mathrm{b}}$, Ramesh Singh ${ }^{\mathrm{d}}$, Jonathan Montomoli ${ }^{\mathrm{n}}$, Michele Mondino ${ }^{\mathrm{a}}$, Barry Greenberg ${ }^{\mathrm{b}}$, Claudio F. Russo ${ }^{\mathrm{a}}$, Victor Pretorius ${ }^{\mathrm{b}}$, Klein Liviu ${ }^{\mathrm{l}}$, Maria Frigerio ${ }^{\mathrm{a}}$, Eric D. Adler ${ }^{\mathrm{b}, * *}$

\section{A R T I C L E I N F O}

\section{Article history:}

Received 19 April 2020

Received in revised form 28 June 2020

Accepted 8 September 2020

Available online $\mathrm{xxxx}$

\section{Keywords:}

Continuous flow left ventricular assist device

Heart transplantation

Outcome

Donor heart

Heart transplant list

Bridge to transplantation

\begin{abstract}
A B S T R A C T
Background: Geographic variations in management and outcomes of individuals supported by continuous-flow left ventricular assist devices (CF-LVAD) between the United States (US) and Europe (EU) is largely unknown. Methods: We created a retrospective, multinational registry of 524 patients who received a CF-LVAD (either HVAD or Heartmate II) between January 2008 and April 2017. Follow up spanned from date of CF-LVAD implant to post-HTx period with a median follow up of 44.8 months.

Results: The cohort included 299 (57.1\%) EU and 225 (42.9\%) US patients. Although the US cohort was significantly older with a higher prevalence of comorbidities, survival was similar between the cohorts (US 63.1\%, EU $68.4 \%$ at 5 years, unadjusted $\log$-rank test $\mathrm{p}=0.43$ ).Multivariate analyses suggested that older age, higher body mass index, elevated creatinine, use of temporary mechanical circulatory support prior CF-LVAD, and implantation of HVAD were associated with increased mortality. Among CF-LVAD patients undergoing HTx, the median time on CF-LVAD support was shorter in the US, meanwhile US donors were younger. Finally, the pattern of adverse events (stroke, gastrointestinal bleedings, late right ventricular failure, and driveline infection) during support differed significantly between US and EU.

Conclusions: Although waitlisted patients in the US on CF-LVAD have higher risk comorbid conditions, the overall outcome is similar in US and EU. Geographic variations with regards to donor characteristics, duration of CFLVAD support prior to transplant, and adverse events on support can explain the disparity in the utilization of mechanical bridge to transplant strategy between US and EU.
\end{abstract}

@ 2020 Elsevier B.V. All rights reserved.

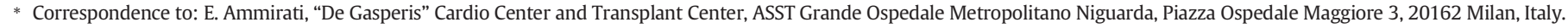

** Correspondence to: E. D. Adler, Department of Cardiology, University of California San Diego, 9500 Gilman Drive, 0613K La Jolla, CA, USA.

E-mail addresses: enrico.ammirati@ospedaleniguarda.it (E. Ammirati), eradler@ucsd.edu (E.D. Adler).

${ }^{1}$ Dr. E. Ammirati and M. Brambatti contributed equally to this work and are co-first authors.
} 


\section{Introduction}

Continuous-flow left ventricular assist devices (CF-LVADs) are commonly used as a bridge therapy to heart transplantation (HTx) [1-3]. Despite the survival benefit, extended time on support increases the rates of CF-LVAD-related complications with a higher risk of delisting or worse post-HTx outcomes [4]. In addition, heart donor availability largely affects duration on support and the subsequent outcomes before and after HTx.

Current large registries (i.e. Interagency Registry for Mechanically Assisted Circulatory Support [INTERMACS], or International Society for Heart and Lung Transplantation [ISHLT] Mechanically Assisted Circulatory Support [IMACS] registries, or European Registry for Patients with Mechanical Circulatory Support [EUROMACS) evaluating CF-LVADs as bridge to HTx do not report survival after HTx $[1,2,5]$. Similarly, the United Network for Organ Sharing (UNOS) only collects data at the time of listing and at the time of HTx, without reporting baseline characteristic at the time of CF-LVAD implant [3]. Hence there is a critical knowledge gap regarding the impact of LVAD on the long term outcomes of patients bridged to transplant.

The United States (US) and Europe (EU) differ in a variety of factors related to CF-LVAD-strategy and HTx indications [6]. Relevant factors that may affect outcomes are numerous, the most obvious being the older age of transplant recipients in the US and older age of transplant donor hearts in the EU. Furthermore, there is a significant discrepancy in HTx and CF-LVAD volume between the US and EU, with the number of CF-LVAD implants being nearly 4 times greater in the US (1700/year in the US compared with 430/year in EU) [7], even though the EU has more than twice the population of the US ( 741 million compared to 328 million respectively). The extent to which geographic variations affect the management and outcomes of individuals who receive a CFLVAD implantation while listed or eventually become listed for transplant is largely unknown.

To address the issues above, we created the TRans-Atlantic registry on VAd and TrAnsplant (TRAViATA), a retrospective, observational, multinational registry that includes CF-LVAD patients who are candidates for HTx or heart-kidney transplant. Herein, we performed an analysis of TRAViATA with the following objectives: (1) characterize overall outcomes from the time of CF-LVAD implant to the posttransplant period, focusing on survival and adverse events, while controlling for regional variations. (2) describe differences in preimplant patients' characteristics, as well as in donors' aspects, between US and EU.

\section{Methods}

\subsection{Participant centers}

Patients in 7 EU hospitals (Niguarda Hospital in Milan, Italy [ $n=72$ ]; Sant'Orsola Malpighi Hospital in Bologna, Italy [n $=7$ ]; Skåne University Hospital in Lund, Sweden [ $\mathrm{n}=35$ ]; Erasmus MC Thoraxcenter in Rotterdam, the Netherlands [ $\mathrm{n}=54]$; Antwerp University Hospital in Antwerp, Belgium [ $\mathrm{n}=22$ ]; University Hospitals Leuven in Leuven, Belgium [ $\mathrm{n}=99$ ]; and University Hospital Centre in Zagreb, Croatia $[\mathrm{n}=10]$ ) and 3 US centers (University of California San Diego, La Jolla, California [ $\mathrm{n}=69$ ]; Inova Heart and Vascular Institute, Falls Church, Virginia [ $\mathrm{n}=88$ ]; and University of California San Francisco, San Francisco, California [ $\mathrm{n}=68]$ ) participated in the TRAViATA registry (Fig. 1A). All the participating sites were required to meet the following criteria: 1) expertise in mechanical circulatory support (MCS) and HTx; 2) active HTx and CF-LVAD programs during the study period; 3) willingness to volunteer, as no funding support for data collection was provided. Institution review board at each respective institution approved the study and, included a waiver of informed consent due to the retrospective nature of the registry.

\subsection{Patient population}

Consecutive patients that received a CF-LVAD in accordance with the study protocol were included. Inclusion criteria for TRAViATA consisted of: (1) age $\geq 16$ years; (2) CF-LVAD implantation between January 2008 and April 2017; (3) implantation of either HeartWare HVAD (HVAD, Minnesota, MN, US) or Heartmate II (HMII, Abbott, Pleasanton, CA, US); (4) listing at any point for HTx or heart and kidney transplantation while supported with CF-LVAD. Exclusion consisted of: (1) patients implanted with HeartMate 3 device (HM3,Abbott Pleasanton, CA, US) as it was still under investigation in the US during the study period; (2) patients treated with bi-ventricular VAD (BiVAD) or total artificial heart (TAH); (3) patients never listed for HTx; (4) prior HTx before CFLVAD implantation. Patient selection and post-operative management were left at the discretion of the local investigators. Last date of data collection in the follow up was March 31, 2018. Median follow-up time on CF-LVAD support was 354 days (first to third quartiles [Q1-Q3]: 168-697) while overall median follow-up time, including both CF-LVAD and HTx was 979 days (Q1-Q3: 448-1669).

\subsection{Data collection and management}

Baseline demographics, prior history of cardiovascular disease, comorbidities, New York Heart Association (NYHA) classification and INTERMACS profile, laboratory values, hemodynamic and echocardiographic parameters were collected. CF-LVAD-related adverse events (i.e. stroke, major bleeding, driveline infections, late right ventricular failure [RVF] and pump thrombosis) were defined using the INTERMACS registry criteria [1]. Survival after HTx and donor characteristics were also collected from each center.

Data were organized using the Research Electronic Data Capture (REDCap), a secure web-based application for building an online database (www.project-redcap.org) managed by O.Ö.B. from Lund University in Lund, Sweden. University of California, San Diego (US) served as the coordinating center, and while the data were not monitored on-site, both E.A. and M.B. checked fidelity of the data and, when needed, contacted local investigators for clarifications. A data dictionary with a detailed description of each variable in the dataset was also provided to each participating center.

\subsection{Statistical analysis}

Continuous variables were presented as either means with standard deviation (SD) or medians with first to third quartile (Q1-Q3). Categorical variables were presented as numbers and percentages. Continuous data were evaluated for normality using the Shapiro-Wilk test. Two-sample t-tests and two-sample Mann Whitney tests were used to compare continuous variables depending on normality, and Fisher's exact tests were used to compare categorical variables. Overall and post-transplant survival of patients receiving CF-LVAD support were estimated using the Kaplan-Meier method. Differences in survival between US and EU were tested using the Mantel log-rank test. Univariate Cox regression analyses were used to determine predictors of overall mortality. We first conducted a univariate an multivariate analysis based on variables for which more than $95 \%$ of values were available for each variables (model 1). Then, we performed a univariate and multivariate analyses also including baseline clinical and diagnostic variables with a larger number of missing data (up to $55 \%$ of missing data for some variables; models 2 and 3). In the model 1, 507 patients with 159 events were included in the multivariate analysis (variables included in the analysis were: age, body mass index [BMI], diabetes, previous sternotomy, history of atrial fibrillation [AF], type of CF-LVAD: HVAD, creatinine, need for temporary MCS, and region of origin: USA). In the model 2 were included baseline variables before CF-LVAD implant that significantly differed between EU and the US cohort (this model included 179 patients and 56 event; and the 

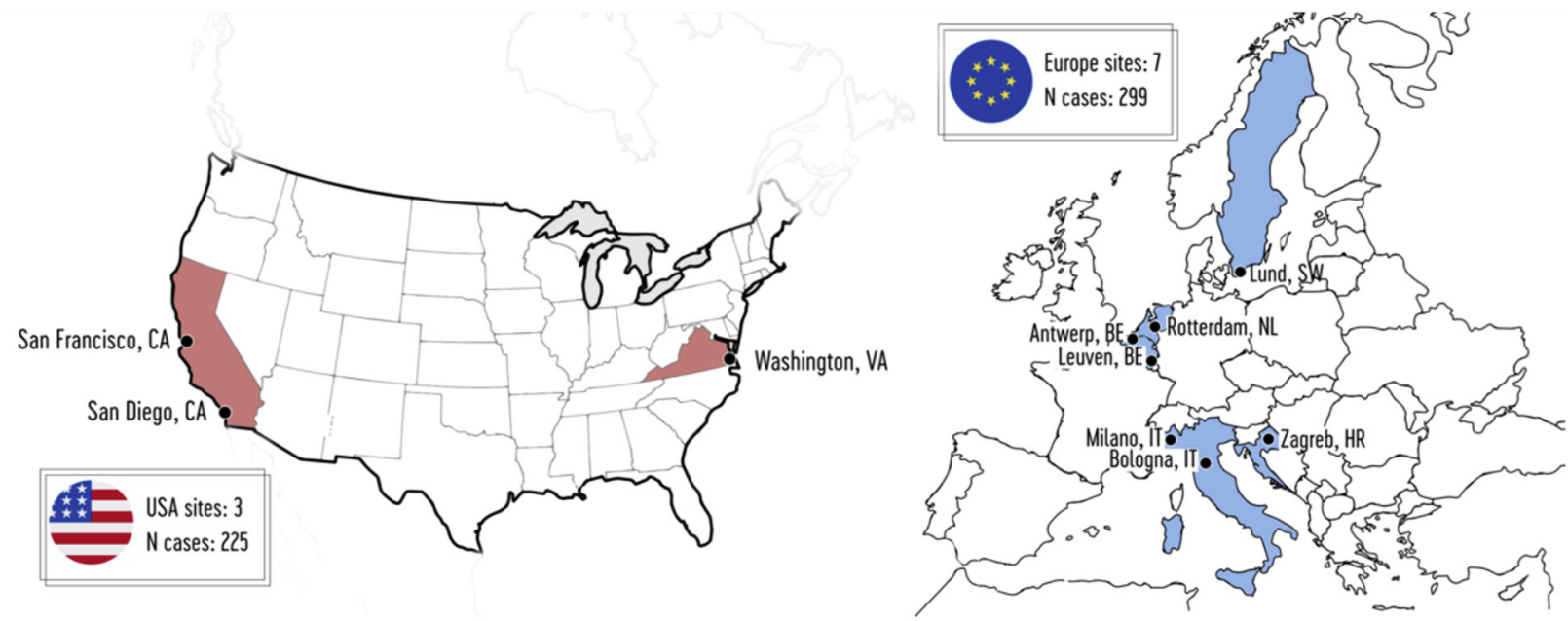

C
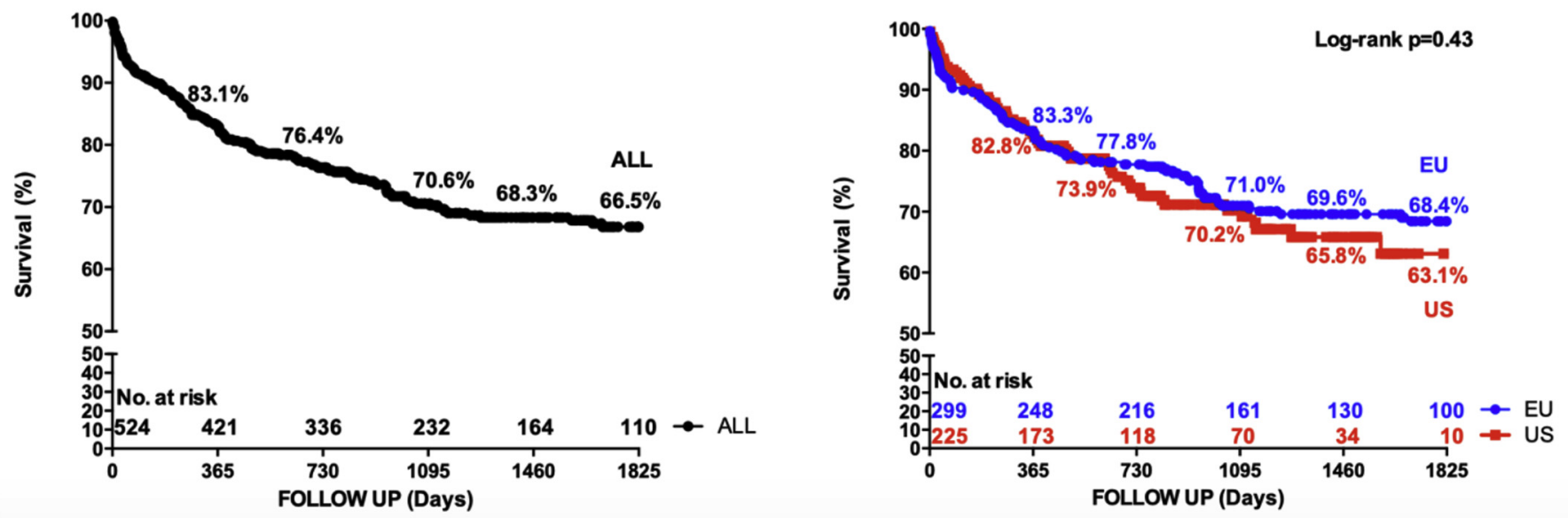

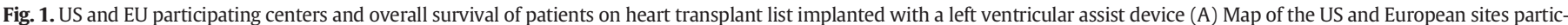

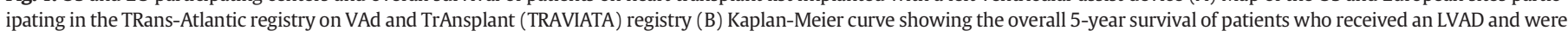

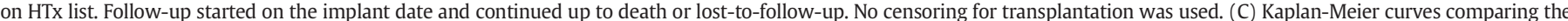

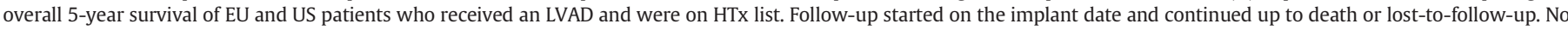
censoring for transplantation was used. Differences in survival were evaluated with the Mantel log-rank test.

following variables: age, BMI, diabetes, history of AF, previous sternotomy, type of CF-LVAD: HVAD, need for temporary MCS, creatinine, right atrial pressure [RAP], mean pulmonary artery mean pressure [mPAP], pulmonary capillary wedge pressure [PCWP], left ventricular ejection fraction [LVEF], left ventricular end-diastolic diameter [LVEDD], tricuspid annular plane systolic excursion [TAPSE], alanine transaminase [ALT], aspartate transaminase [AST] and region of origin: USA. In the model 3 we included the variables included in the model 1 plus variables associated with right ventricular function: RAP and TAPSE (this model included 199 patients and 66 events). Covariates with a pvalue $<0.2$ were used to fit multivariate models, while the region variable (US vs. EU) was forced into the model. Time dependent survival analysis was used to adjust for effect of HTx (treated as timedependent variable) on the overall survival of the patients. Waiting time on CF-LVAD until HTx was calculated treating HTx as the event and censoring the patients who were lost to follow up or dead before the HTx. Adverse events while supported on CF-LVAD were reported as events $/ 100$ patient per month, which was calculated as the number of events divided by the cumulative support durations for all patients. Comparisons of adverse event rates between EU and US occurring in a 3-year follow up period following CF-LVAD implant were performed with a Poisson regression adjusted for differences at baseline (age, body mass index [BMI], diabetes, history of atrial fibrillation [AF], and type of CF-LVAD). Median follow-up time in month was calculated using reverse Kaplan-Meier estimate. All statistical comparisons were 2 -sided, and the significance level was set at $\mathrm{p}=0.05$. Statistical analyses were done using $\mathrm{R}$ programming language and environment (version 3.6.1, R Foundation for Statistical Computing, Vienna, Austria) and GraphPad Prism (version 6, GraphPad Software Inc., CA, US).

\section{Results}

\subsection{Baseline patient characteristics at the time of CF-LVAD implantation}

A total of 524 patients ( 225 from US and 299 from EU) were included in the TRAViATA registry. Patients were predominantly male $(84.4 \%)$ with a median age of 55 years (Q1-Q3, 45-61). Table 1 shows the distribution of baseline characteristics from both US and EU cohorts. Notable differences include a more racially diverse and significantly older cohort in the US compared with EU cohort. The US cohort also had a 
significantly higher prevalence of comorbidities, including obesity, diabetes and $\mathrm{AF}$.

Severity of illness at the time of CF-LVAD implantation as determined by INTERMACS class 1 or $2(43.9 \%$ vs. $47.3 \%, \mathrm{p}=0.48)$ and the need for temporary MCS (25.8\% vs. $30.9 \%, \mathrm{p}=0.24)$ were similar between US and EU cohorts. However, the axial HMII device was implanted more frequently in the EU cohort (71.9\%), while the centrifugal Heartware HVAD device was more common in the US cohort (56.0\%; $\mathrm{p}<0.001$ ). The median follow-up in the EU cohort was longer compared with the US cohort (60.0 vs. 34.0 months) even if it was not statistically significant based on the log rank test (Table 1 ).

\subsection{Outcomes and predictors of mortality}

Overall patient survival at 1 year was $83.1 \%$ and at 5 years was $66.5 \%$ estimated by the Kaplan-Meier curves (Fig. 1B) and was similar between the two cohorts (US 63.1\% vs. EU 68.4\% at 5 years, unadjusted Mantel log-rank test $\mathrm{p}=0.43$; Fig. $1 \mathrm{C}$ ). Fig. 2 shows the status of patients that reached a complete follow up at 1,2 , and 3 years. The overall proportion of patients alive and still on mechanical support at 1,2 , and 3 years was 50.2\% (256/510), 28.1\% (128/456) and 13.8\% (52/378) respectively. There were no significant differences among US and EU cohorts in the proportion of this status. At 1-year patients that were transplanted in the US cohort was $46.0 \%$ vs. $33.8 \%$ in EU ( $p=0.11$ ). None of the patients in the registry recovered to the point where CF-LVAD was explanted. Supplemental Fig. 1 shows the status (alive or dead) of patients that reached a complete follow up at 1,2, and 3 years. We observed a larger proportion of death in the US group compared the EU group among patients that complete at 3 year follow up ( $47.0 \%$ vs. $34.0 \%$ respectively, $p=0.013$ ), even if this result must be affected by the fact that a larger proportion of US patients, that could survive beyond 3 years, did not reach a 3 years of follow-up compared with the EU cohort ( $40.4 \%$ vs. $18.4 \%$ respectively, $\mathrm{p}=0.001$ ).

Supplemental Table 1 shows univariate analysis of baseline factors before CF-LVAD associated with the occurrence of death. Table 2 shows the univariate and multivariate Cox regression analyses evaluating predictors of mortality in the overall cohort. In the adjusted model, independent predictors of overall mortality were older age (hazard ratio [HR] 1.038, 95\%CI 1.020-1.057), higher BMI (HR 1.051, 95\%CI 1.016-1.087), higher creatinine (HR 1.221, 95\%CI 1.021-1.460), temporary MCS (HR 1.487, 95\%CI 1.062-2.081) before CF-LVAD implantation and use of Heartware HVAD (vs. HMII; HR of 1.549 ; $95 \% \mathrm{CI}$ 1.105-2.172). Origin (US vs. EU) did not emerge as a variable associated with survival. Both the interactions terms Region*HTx (HR 1.055; 95\%CI $0.530-2.102$, p value $=0.879)$ and Region*type of device (HR 0.664; $95 \% \mathrm{CI} 0.371-1.188$, $\mathrm{p}$ value $=0.168$ ) were tested and both were not significant.

When HTx was added in the model as time dependent covariate, elder age, higher BMI, increased creatinine, pre-CF-LVAD use of a temporary MCS and implantation of HVAD (vs. HMII) remained independent factors associated with mortality. HTx was independently associated with an improved survival rate (HR 0.456, 95\%CI 0.308-0.675), and US origin emerged as a variable independently associated with better survival (HR 0.708, 95\%CI 0.510-0.983) (Table 2). Multivariate model analysis that included baseline variables before CF-LVAD implant that significantly differend between EU and the US cohort (model 2; Supplemental Table 2) shows in the adjusted model, independent predictors of overall mortality were older age (HR 1.047, 95\%CI 1.010-1.084), higher BMI (HR 1.071, 95\%CI 1.004-1.142), and previous sternotomy (HR 2.113, 95\%CI 1.048-4.260), whereas borderline significance was observed for use of Heartware HVAD (vs. HMII; HR of 1.804; $95 \% \mathrm{CI} 0.940-3.461, \mathrm{p}=0.076$ ), and higher TAPSE (HR 0.528; 95\%CI $0.270-1.034 ; \mathrm{p}=0.063$ ). Multivariate model analysis that included variables in model 1 plus variables associated with right ventricular function (RAP and TAPSE; called model 3, Supplemental Table 3) shows in the adjusted model, independent predictors of overall mortality were older age (HR 1.041, 95\%CI 1.009-1.073), and use of Heartware HVAD (vs. HMII; HR of 1.794; 95\%CI 1.06-3.198), whereas borderline significance was observed for higher BMI (HR 1.051, 95\%CI 0.991-1.115; $\mathrm{p}=0.098$ ), previous sternotomy (HR 1.869, 95\%CI 0.975-3.198; $\mathrm{p}=$ 0.060 ), and higher TAPSE (HR 0.564; 95\%CI 0.308-10.30; $\mathrm{p}=0.062$ ). Origin (US vs. EU) did not emerge as a variable associated with survival also in model 2 and 3 of multivariate analyses.

\subsection{Time to HTx and donor characteristics}

We analyzed time with CF-LVAD until HTx, and donor characteristics to further understand the effect of the origin in patients mechanically bridged to HTx in US vs. EU. The proportion of patients that were on HTx list at the time of CF-LVAD implantation (BTT indication) was similar in the US cohort and the EU cohort (52.9\% vs. $55.2 \%$ respectively; $\mathrm{p}=0.66$ ). On average patients were listed for HTx the same day they received a CF-LVAD both in US and the EU (Fig. 3A). The cause-specific hazard of HTx within US patients was 13.3\% higher compared with those in EU cohort, but the difference was not statistically significant $(\mathrm{p}=0.29$ ). Supplemental Table 4 compares the baseline characteristics of patients with CF-LVAD who received and who did not undergo HTx. Among patients who received HTx, the median time on CF-LVAD support was shorter among those in the US cohort compared to those in the EU cohort ( 238 vs. 342 days, respectively; $\mathrm{p}=0.0003$, Fig. 3B).

Supplemental Table 5 summarizes the donor characteristics in the US and EU cohorts. Donors were significantly younger in the US cohort compared with the EU cohort (median age of 29 years; Q1-Q3, 23-39 vs. 48 years; $\mathrm{Q} 1-\mathrm{Q} 3,38-54$, respectively, $\mathrm{p}<0.0001$, Fig. $3 \mathrm{C}$ ). Donors in the US cohort were more likely to have been resuscitated from cardiac arrest compared with EU donors ( $\mathrm{p}<0.0001$ ). There were no significant differences in the use of sex-mismatched donors, while utilization of undersized donors (defined as a donor-to-recipient weight ratio of 0.80 or less) was more common in the US cohort compared with the EU cohort ( $p=0.011$ ). Ischemic time, donor LVEF, and donor inotrope requirement were not different between the two groups. Finally, the post-transplant survival was similar between the two cohorts (US $82.0 \%$ vs. EU $84.7 \%$ at 4 years, unadjusted Mantel log-rank test $\mathrm{p}=$ 0.99; Fig. 3D). Univariate and multivariate Cox regression analyses of factors associated with the occurrence of death in post-transplant patients are shown in Supplemental Table 6. Increased age (HR 1.06, 95\% CI 1.03-1.10; $>0.001$ ) and Heartware HVAD implant (HR 2.08, 95\% CI $1.06-4.09, \mathrm{p}=0.03$ ) are the only variables independently associated with post-transplant mortality. On the other hand, origin from USA had a borderline protective effect on post transplant mortality (HR 0.43, 95\% CI 0.18-1.02, $\mathrm{p}=0.054)$. Supplemental Table 7 reports the causes of death during CF-LVAD in the US and EU cohort, where cerebrovascular accidents was the main cause of death; and Supplemental Table 8 reports the causes of death after HTx in the US and EU groups, where graft failure followed by sepsis were the main causes of death in both.

\subsection{Adverse events on CF-LVAD support}

Supplemental Table 9 shows the number of patients with CF-LVAD related adverse events within 3 years of implantation, and the incident rate of these events. Poisson regression analysis showed a significant difference in the incidence rates of overall stroke (both ischemic and hemorrhagic), ischemic stroke (including transient ischemic attack [TIA]), gastrointestinal bleedings (GIBs), late RVF, and driveline infection in the US and EU cohort. Incidence rates of hemorrhagic stroke and pump thrombosis were not significantly different between the US and EU cohorts. Supplemental Table 10 summarizes the factors associated with CF-LVAD adverse events in the multivariate analysis. The factors independently associated with stroke were the age at implant and implant of HVAD. Variables independently associated with GIB were US origin (incidence rate ratio [IRR] 2.604, 95\%CI 1.793-3.810), elderly age, and the implantation of HMII. Development of late RVF was 
Table 1

Clinical presentation and diagnostic findings in 524 patients on heart transplant list treated with continuous-flow left ventricular assist device (CF-LVAD).

\begin{tabular}{|c|c|c|c|c|c|c|c|}
\hline \multirow[t]{3}{*}{ Characteristics } & \multirow{2}{*}{\multicolumn{2}{|c|}{ Overall }} & \multicolumn{4}{|c|}{ Continents } & \multirow[t]{3}{*}{ p-value } \\
\hline & & & \multicolumn{2}{|l|}{ EU } & \multicolumn{2}{|l|}{ US } & \\
\hline & Data (n.) & Value & Data (n.) & Value & Data (n.) & Value & \\
\hline \multicolumn{8}{|l|}{ Demographics \& comorbidities } \\
\hline Age, years, median (Q1-Q3) & 524 & $55(45-61)$ & 299 & $53(44-59)$ & 225 & $59(50-64)$ & $<0.001$ \\
\hline Age $\geq 60$ years, $n(\%)$ & 524 & $173(33.0)$ & 299 & $69(23.1)$ & 225 & $104(46.2)$ & $<0.001$ \\
\hline Male, $\mathrm{n}(\%)$ & 524 & $442(84.4)$ & 299 & $252(84.3)$ & 225 & $190(84.4)$ & 1 \\
\hline Race hispanic, n (\%) & 523 & $36(6.9)$ & 299 & $2(0.7)$ & 224 & $34(15.2)$ & $<0.001$ \\
\hline Race, n (\%) & 521 & & 299 & & 222 & & $<0.001$ \\
\hline Asian & & $25(4.8)$ & & $9(3.0)$ & & $16(7.2)$ & \\
\hline Black & & $59(11.3)$ & & $6(2.0)$ & & $53(23.9)$ & \\
\hline Caucasian & & $393(75.4)$ & & $282(94.3)$ & & $111(50.0)$ & \\
\hline Others & & $44(8.4)$ & & $2(0.7)$ & & $42(18.9)$ & \\
\hline Blood type, n (\%) & 524 & & 299 & & 225 & & 0.327 \\
\hline A & & $201(38.4)$ & & $124(41.5)$ & & $77(34.2)$ & \\
\hline $\mathrm{AB}$ & & $18(3.4)$ & & $11(3.7)$ & & $7(3.1)$ & \\
\hline B & & $70(13.4)$ & & $36(12.0)$ & & $34(15.1)$ & \\
\hline 0 & & $235(44.8)$ & & $128(42.8)$ & & $107(47.6)$ & \\
\hline BMI, kg/m², median (Q1-Q3) & 521 & $25.4(22.7-29.3)$ & 296 & $24.6(22.5-27.9)$ & 225 & $26.9(23.4-31.4)$ & $<0.001$ \\
\hline $\mathrm{BMI} \geq 30 \mathrm{~kg} / \mathrm{m}^{2}, \mathrm{n}(\%)$ & 521 & $113(21.7)$ & 296 & $42(14.2)$ & 225 & $71(31.6)$ & $<0.001$ \\
\hline Diabetes mellitus, $\mathrm{n}(\%)$ & 524 & $131(25.0)$ & 299 & $39(13.0)$ & 225 & $92(40.9)$ & $<0.001$ \\
\hline Insulin dependent diabetes, n (\%) & 465 & $64(13.8)$ & 240 & $20(8.3)$ & 225 & $44(19.6)$ & $<0.001$ \\
\hline History of atrial fibrillation, $\mathrm{n}(\%)$ & 522 & $162(31.0)$ & 297 & $76(25.6)$ & 225 & $86(38.2)$ & 0.002 \\
\hline INTERMACS class $1-2, \mathrm{n}(\%)$ & 521 & $239(45.9)$ & 298 & $141(47.3)$ & 223 & $98(43.9)$ & 0.478 \\
\hline Ischemic cause of $\mathrm{HF}, \mathrm{n}(\%)$ & 524 & $228(43.5)$ & 299 & $135(45.2)$ & 225 & $93(41.3)$ & 0.423 \\
\hline $\mathrm{ICD}, \mathrm{n}(\%)$ & 524 & $239(45.6)$ & 299 & $119(39.8)$ & 225 & $120(53.3)$ & 0.002 \\
\hline CRT, n (\%) & 524 & $139(26.5)$ & 299 & $86(28.8)$ & 225 & $53(23.5)$ & 0.216 \\
\hline \multicolumn{8}{|l|}{ Hemodynamics } \\
\hline RAP, mmHg, median (Q1-Q3) & 382 & $10(6-15)$ & 168 & $9(5-14)$ & 214 & $11(8-15)$ & 0.006 \\
\hline mPAP, mmHg, median (Q1-Q3) & 385 & $36(28-42)$ & 176 & $36(28-42)$ & 209 & $35(30-42)$ & 0.832 \\
\hline PCWP, mmHg, median (Q1-Q3) & 379 & $25(20-30)$ & 170 & $25(20-31)$ & 209 & $25(20-30)$ & 0.328 \\
\hline $\mathrm{CI}, \mathrm{L} / \mathrm{min} / \mathrm{m}^{2}$, median $(\mathrm{Q} 1-\mathrm{Q} 3)$ & 351 & $1.8(1.5-2.2)$ & 142 & $1.7(1.4-2.0)$ & 209 & $1.9(1.5-2.3)$ & 0.002 \\
\hline PVR, WU, median (Q1-Q3) & 322 & $2.9(1.8-4.2)$ & 138 & $3.1(2.0-4.2)$ & 184 & $2.7(1.6-4.2)$ & 0.140 \\
\hline \multicolumn{8}{|l|}{ Echocardiographic parameters } \\
\hline LVEF \%, median (Q1-Q3) & 471 & $20(15-25)$ & 247 & $20(15-25)$ & 224 & $18(14-23)$ & $<0.001$ \\
\hline LVEDD, cm, median (Q1-Q3) & 457 & $6.9(6.1-7.6)$ & 262 & $6.9(6.1-7.6)$ & 195 & $6.9(6.2-7.6)$ & 0.761 \\
\hline TAPSE, cm, median (Q1-Q3) & 239 & $1.5(1.3-1.7)$ & 152 & $1.5(1.3-1.7)$ & 87 & $1.5(1.2-1.8)$ & 0.623 \\
\hline \multicolumn{8}{|l|}{ Laboratory values } \\
\hline Creatinine, mg/dL, median (Q1-Q3) & 523 & $1.3(1.0-1.6)$ & 298 & $1.3(1.0-1.6)$ & 225 & $1.2(1.0-1.7)$ & 0.877 \\
\hline Bilirubin, mg/dL, median (Q1-Q3) & 453 & $1.1(0.7-1.7)$ & 228 & $1.0(0.6-1.6)$ & 225 & $1.2(0.7-1.8)$ & 0.069 \\
\hline AST, UI/L, median (Q1-Q3) & 451 & $28(21-44)$ & 226 & $25(18-43)$ & 225 & $30(22-44)$ & 0.006 \\
\hline ALT, UI/L, median (Q1-Q3) & 454 & $28(18-50)$ & 229 & $26(16-52)$ & 225 & $29(19-48)$ & 0.120 \\
\hline \multicolumn{8}{|l|}{ Surgical aspects } \\
\hline Previous sternotomy, n (\%) & 524 & $83(15.8)$ & 299 & $41(13.7)$ & 225 & $42(18.7)$ & 0.147 \\
\hline BTT at time of implant, $\mathrm{n}(\%)$ & 524 & $499(95.2)$ & 299 & $298(99.7)$ & 225 & $201(89.3)$ & $<0.001$ \\
\hline Need for MCS pre-implant, n (\%) & 513 & $147(28.7)$ & 288 & $89(30.9)$ & 225 & $58(25.8)$ & 0.238 \\
\hline HeartMate II device, n (\%) & 524 & $314(59.9)$ & 299 & $215(71.9)$ & 225 & $99(44.0)$ & $<0.001$ \\
\hline Heartware HVAD device $\mathrm{n}(\%)$ & 524 & $210(40.1)$ & 299 & $84(28.1)$ & 225 & $126(56.0)$ & $<0.001$ \\
\hline Concurrent surgery, n (\%) & 517 & $127(20.1)$ & 292 & $29(9.9)$ & 225 & $75(33.3)$ & $<0.001$ \\
\hline Minimal invasive surgery, n (\%) & 524 & $58(11.1)$ & 299 & $14(4.7)$ & 225 & $44(19.6)$ & $<0.001$ \\
\hline OF cannula in descending aorta, $\mathrm{n}(\%)$ & 524 & $14(2.7)$ & 299 & $8(2.7)$ & 225 & $6(2.7)$ & 1 \\
\hline \multicolumn{8}{|l|}{ In-hospital antiplatelet therapy } \\
\hline Use of aspirin, $n(\%)$ & 443 & $377(85.1)$ & 269 & $219(81.4)$ & 174 & $158(90.8)$ & 0.006 \\
\hline \multicolumn{8}{|l|}{ Dosage of aspirin } \\
\hline$\leq 100 \mathrm{mg}, \mathrm{n}(\%)$ & 377 & $241(63.9)$ & 219 & $194(88.6)$ & 158 & $47(29.7)$ & $<0.001$ \\
\hline$\geq 200$ mg, n (\%) & 377 & $136(36.1)$ & 219 & $25(11.4)$ & 158 & $111(70.3)$ & $<0.001$ \\
\hline Use of dipyridamole, n (\%) & & & 266 & $70(26.3)$ & 214 & $68(31.8)$ & 0.22 \\
\hline Use of another antiplatelet, $\mathrm{n}(\%)$ & 479 & $36(7.5)$ & 265 & $21(7.9)$ & 214 & $15(7.0)$ & 0.73 \\
\hline Last in-hospital INR & 452 & $2.4(2.2-2.6)$ & 246 & $2.4(2.2-2.8)$ & 206 & $2.3(2.1-2.5)$ & $<0.001$ \\
\hline Last in-hospital LDH & 385 & $303(230-423)$ & 184 & $320(221-520)$ & 201 & $294(235-386)$ & 0.21 \\
\hline Follow up Time, months, median (Q1-Q3) & 524 & $44.8(28.2-68.1)$ & 299 & $60.0(55.0-65.1)$ & 225 & $34.0(29.6-39.2)$ & $0.326^{*}$ \\
\hline
\end{tabular}

P-values in bold report the statistically significant differences.

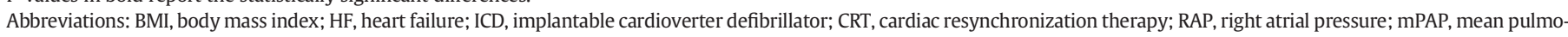

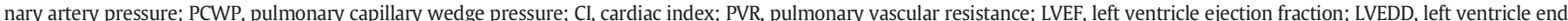

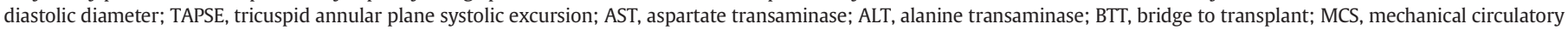
support; OF, outflow; INR, international normalized ratio; LDH, lactate dehydrogenase; WU, Woods Units.

* Median follow up time in month was calculated using Kaplan-Meier based method.

independently associated with US origin (IRR 4.509, 95\%CI 2.971-7.011), diabetes and implantation with an HVAD. Driveline infection was associated with diabetes, and HVAD implant, whereas US origin (IRR 0.318, 95\%CI 0.228-0.438) was associated with decreased risk in the multivariate analysis. Finally, pump thrombosis was independently associated with older age and elevated BMI at implant. Unexpectedly, diabetes was associated with a lower rate of pump thrombosis. 

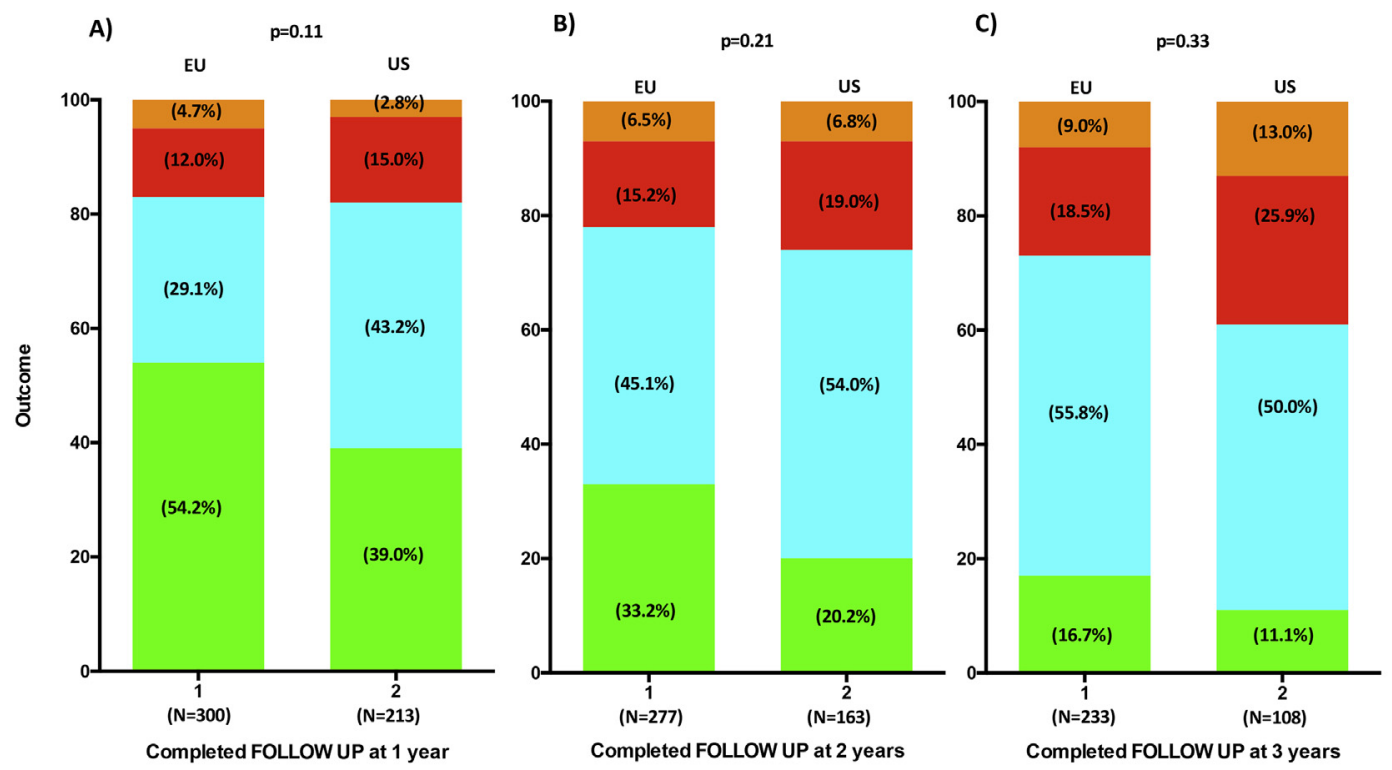

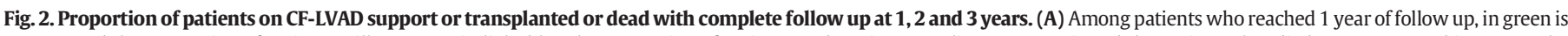

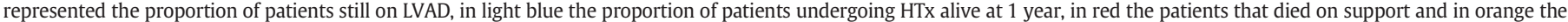

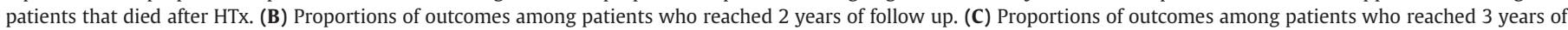

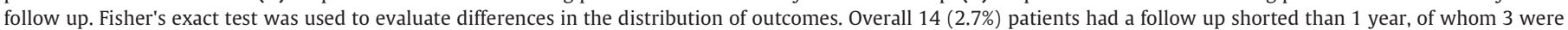

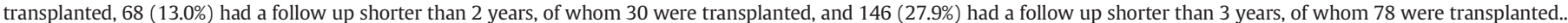
(For interpretation of the references to colour in this figure legend, the reader is referred to the web version of this article.)

Table 2

Univariate and multivariate Cox regression analysis of factors associated with the occurrence of death in patients with left ventricular assist devices including geographical origin.

\begin{tabular}{|c|c|c|c|c|c|c|}
\hline \multirow[t]{3}{*}{ Variables } & \multirow{2}{*}{\multicolumn{3}{|c|}{$\begin{array}{l}\text { US }(\mathrm{n}=225) \text { vs. EU } \\
(\mathrm{n}=299) \\
\text { Univariate analysis }\end{array}$}} & \multirow{2}{*}{\multicolumn{3}{|c|}{$\begin{array}{l}\text { US }(\mathrm{n}=225) \text { vs. EU } \\
(\mathrm{n}=299) \\
\text { Multivariate analysis }\end{array}$}} \\
\hline & & & & & & \\
\hline & HR & $95 \% \mathrm{CI}$ & $\mathrm{p}$-value & HR & $95 \% \mathrm{CI}$ & $\mathrm{p}$-value \\
\hline Age & 1.037 & $1.021-1.053$ & $<0.001$ & 1.038 & $1.020-1.057$ & $<0.001$ \\
\hline BMI & 1.043 & $1.012-1.075$ & 0.006 & 1.051 & $1.016-1.087$ & 0.004 \\
\hline Diabetes & 1.650 & $1.190-2.289$ & 0.003 & 1.323 & $0.909-1.926$ & 0.144 \\
\hline $\begin{array}{l}\text { Previous } \\
\text { sternotomy }\end{array}$ & 1.490 & $1.026-2.164$ & 0.036 & 1.176 & $0.798-1.734$ & 0.413 \\
\hline $\begin{array}{l}\text { HeartWare HVAD } \\
\text { device }\end{array}$ & 1.500 & $1.089-2.065$ & 0.013 & 1.549 & $1.105-2.172$ & 0.011 \\
\hline Creatinine & 1.233 & $1.048-1.450$ & 0.012 & 1.221 & $1.021-1.460$ & 0.029 \\
\hline Need for t-MCS & 1.280 & $0.920-1.782$ & 0.143 & 1.487 & $1.062-2.081$ & 0.021 \\
\hline Region: USA & 1.172 & $0.854-1.610$ & 0.326 & 0.706 & $0.488-1.022$ & 0.065 \\
\hline \multicolumn{2}{|c|}{ Adjusted for effect of HTx } & \multicolumn{5}{|c|}{ Multivariate analysis } \\
\hline \multicolumn{2}{|l|}{ Variables } & \multicolumn{2}{|l|}{ HR } & \multicolumn{2}{|l|}{$95 \% \mathrm{CI}$} & p-value \\
\hline \multicolumn{2}{|l|}{ Age } & \multicolumn{2}{|l|}{1.041} & \multicolumn{2}{|c|}{$1.025-1.058$} & $<0.001$ \\
\hline \multicolumn{2}{|l|}{ BMI } & \multicolumn{2}{|l|}{1.042} & \multicolumn{2}{|c|}{$1.012-1.074$} & 0.006 \\
\hline \multicolumn{2}{|l|}{ Diabetes } & \multicolumn{2}{|l|}{1.171} & \multicolumn{2}{|c|}{$0.841-1.631$} & 0.350 \\
\hline \multicolumn{2}{|l|}{ Previous sternotomy } & \multicolumn{2}{|l|}{1.244} & \multicolumn{2}{|c|}{$0.889-1.741$} & 0.202 \\
\hline \multicolumn{2}{|c|}{ HeartWare HVAD device } & \multicolumn{2}{|l|}{1.467} & \multicolumn{2}{|c|}{$1.090-1.975$} & 0.011 \\
\hline \multicolumn{2}{|c|}{ Creatinine } & \multicolumn{2}{|l|}{1.244} & \multicolumn{2}{|c|}{$1.064-1.454$} & 0.006 \\
\hline \multicolumn{2}{|l|}{ Need for t-MCS } & \multicolumn{2}{|l|}{1.582} & \multicolumn{2}{|c|}{$1.179-2.123$} & 0.002 \\
\hline \multicolumn{2}{|l|}{ Region: USA } & \multicolumn{2}{|l|}{0.708} & \multicolumn{2}{|c|}{$0.510-0.983$} & 0.039 \\
\hline \multicolumn{2}{|l|}{ HTx } & \multicolumn{2}{|l|}{0.456} & \multicolumn{2}{|c|}{$0.308-0.675$} & $<0.001$ \\
\hline
\end{tabular}

P-values in bold report the statistically significant associations.

Abbreviations: HR, hazard ratio; $\mathrm{CI}$, confidential interval; t-MCS, temporary mechanical circulatory support, HTx, heart transplantation.

\section{Discussion}

In summary, we present a comprehensive, multinational, contemporary registry aimed at the comparison of end-stage HF supported with CF-LVAD awaiting transplant in the US and EU. While other studies censored patients at the time of HTx, our study included post-HTx follow up to better evaluate the overall success of the intended therapeutic strategy, and to evaluate if, and how time on MCS affects post-transplant outcomes. Hence, this registry provides a holistic picture of CF-LVAD patients listed for HTx. Our principal finding is that the overall survival in CF-LVAD patients, whether or not they were transplanted, was similar between the US and EU cohorts. In addition, significant differences in baseline characteristics and practice patterns between the US and EU were observed. Patients in the US cohort were older and with more co-morbidities, whereas those in the EU cohort were younger, with lower BMI and less diabetes. In other two multivariate models that included also echocardiographic and hemodynamics data, still US vs. EU origin did not emerge as an independent factor associated with outcome. These models included only $34.2 \%$ (models 2 ) and 38.0\% (model 3 ) of the patients of whole cohort, due to missing data. Based on these subanalysis older age consistenly emerged as associated with increased mortality. Patients from the US are $11.3 \%$ more likely to receive an HTx than EU patients, even if it was not statistically significant $(\mathrm{p}=0.29$ ). Furthermore, EU patients wait longer for transplantation and received older donors. When the variable HTx is added in the multivariate analysis, US origin emerged as a variable associated with a better survival, likely as a result of the abovementioned points.

End-stage HF is unique in that treatment is partially dictated by local availability of donor organs, allocation policies, and healthcare service organizations; hence, wide geographic variability in practice exists between the US and EU. Differences in donor organ availability and their clinical characteristics, including older age in EU than in the US, also impact outcomes in those who undergo CF-LVAD placement as a BTT. Ultimately, these factors may explain some of the differences we observed.

CF-LVAD related adverse events differed significantly between the US and EU cohorts. The incidence of RVF and GIB were higher in the US, while rates of driveline infection were higher in the EU. The higher rates of RVF and GIB observed in the US cohort may be explained by the differences in baseline co-morbidities, primarily older age and diabetes. It is uncertain whether differences in prescribing patterns between the US and Europe may explain these differences as well. It is also unclear why driveline infections were observed more frequently in the EU; further study of these findings is indicated. 
A)

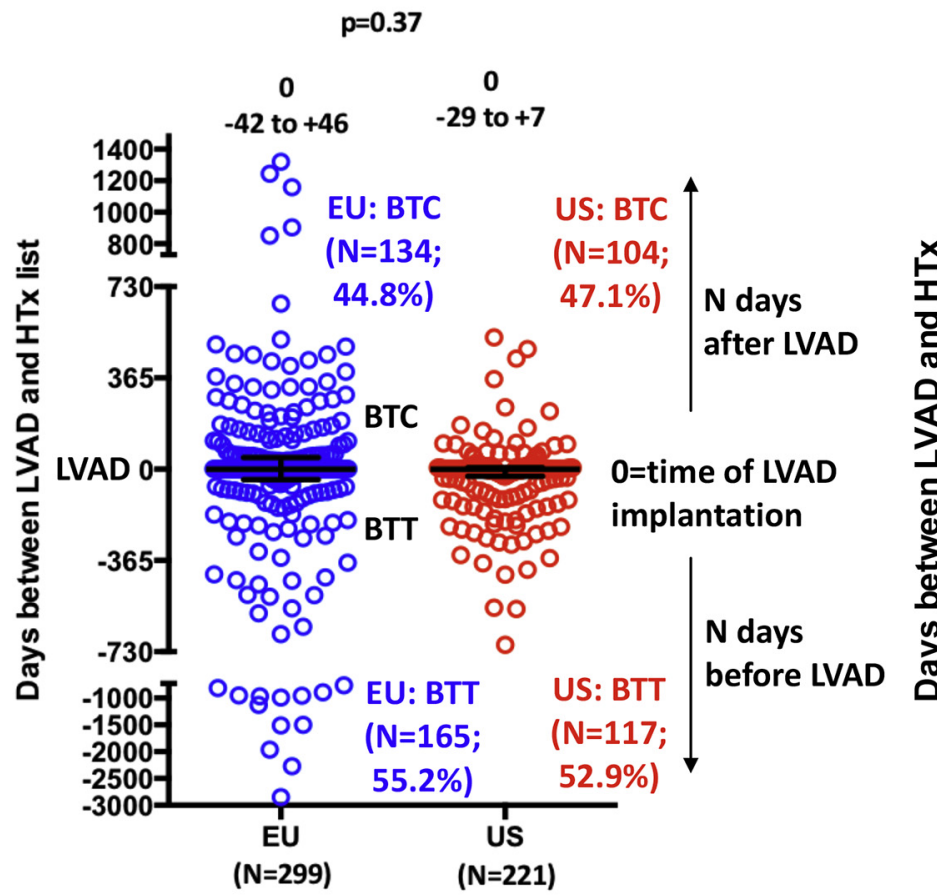

B) $p=0.0003$

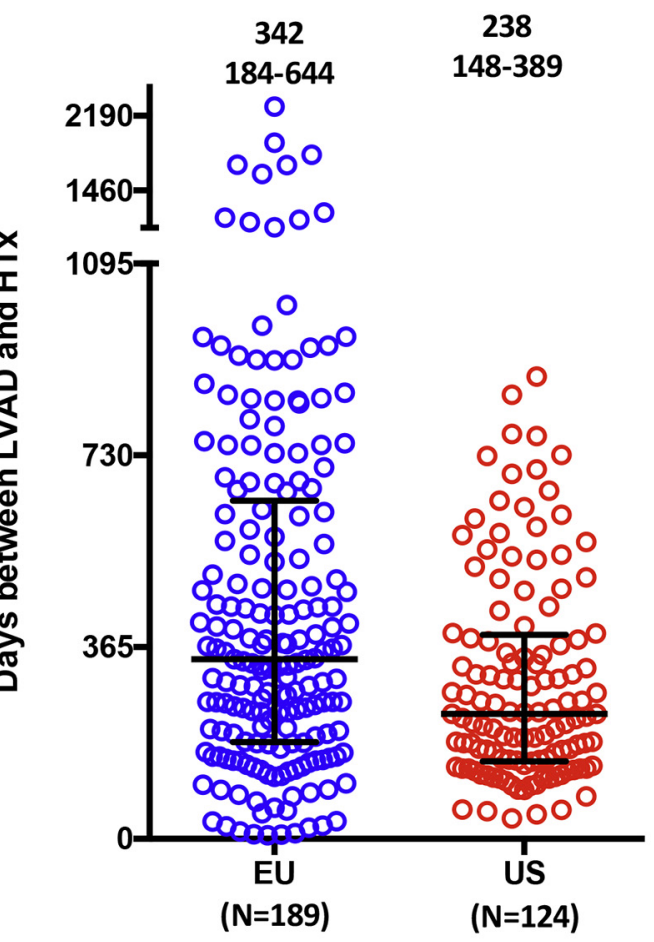

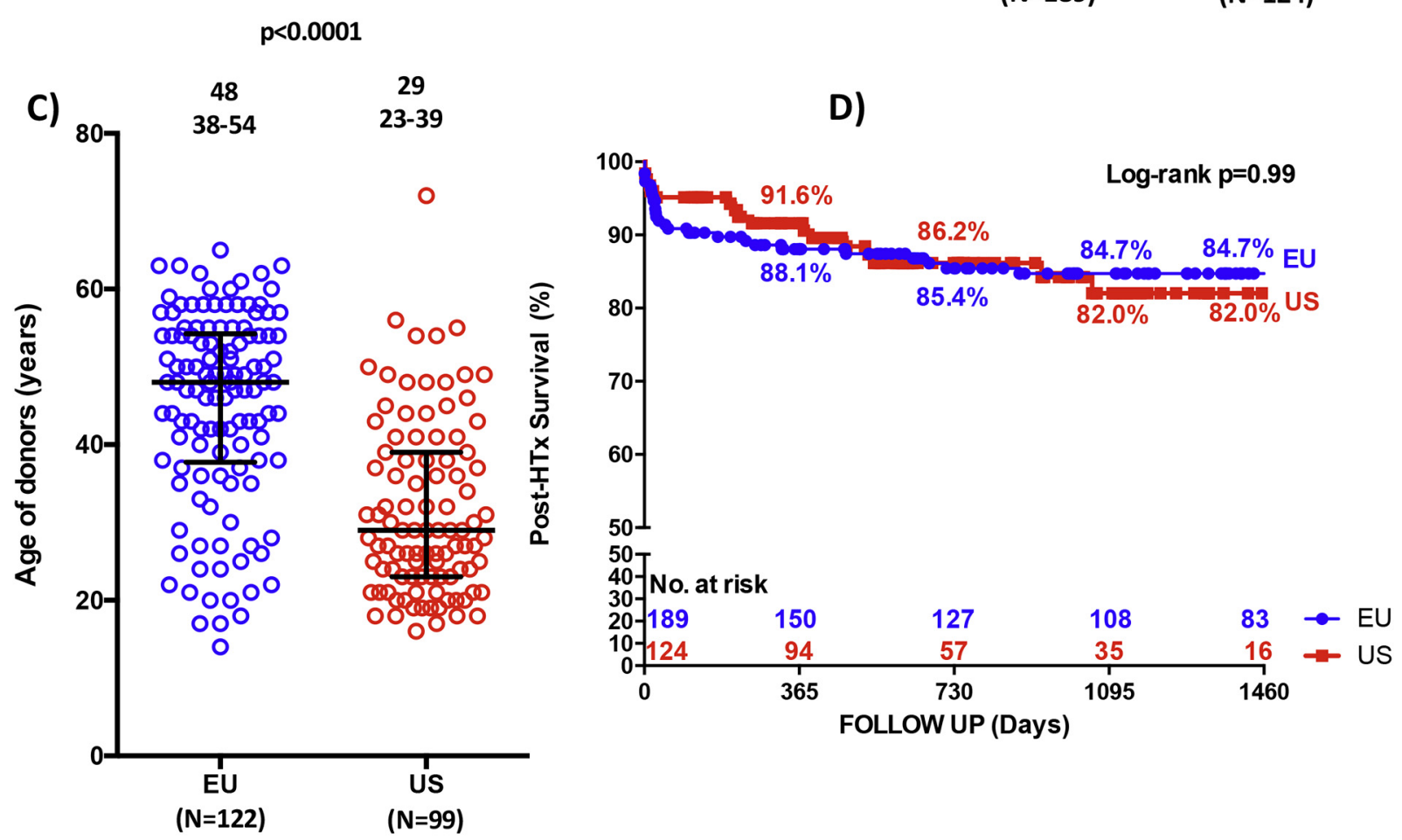

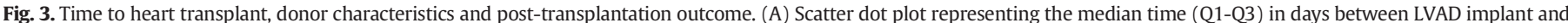

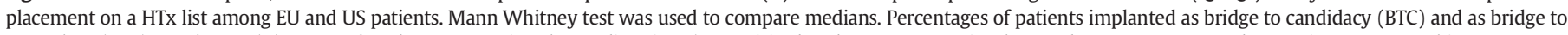

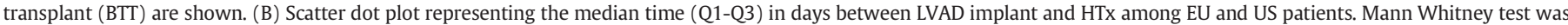

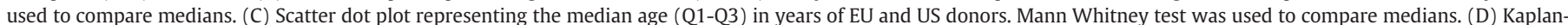

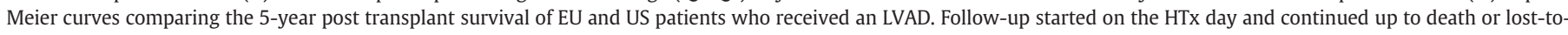
follow-up. Differences in survival were evaluated with the Mantel log-rank test.

Of note, specific subgroups of CF-LVAD patients did worse than others, regardless of geographic location, such as older-age. This suggests performing surgery once in these patients: CF-LVAD as destination therapy or direct to transplant. Though there is some trepidation in transplantation of elderly patients, contemporary studies suggest outcomes can be comparable [8].

Creatinine also emerged as a baseline variable associated with prognosis consistently with other studies, including an extensive analysis of 
UNOS patients that showed impaired renal function predicted waitlist mortality [9]. It remains unclear how to select patients with considerable renal impairment for CF-LVAD, as opposed to listing directly for a multi-organ transplant. A small minority of patients in this registry underwent heart-kidney transplantation, predominantly in the US cohort compared with the EU cohort (4.1 vs. $0.5 \%$, respectively). Increased BMI was also associated with worse outcomes in both the US and EU cohorts. This has historically been shown to be a risk factor for patients that undergo HTx as well $[10,11]$. This may be due to the fact that larger patients may have to wait a longer time to find a suitable donor. It also appears that patients with a high BMI were associated with pump thrombosis, though causation was not clearly established.

Our findings are consistent with a previous study of BTT patients demonstrating that patients over 60 years old, eGFR $<40 \mathrm{~mL} / \mathrm{min} /$ $1.73 \mathrm{~m}^{2}$ and BMI $>30 \mathrm{~kg} / \mathrm{m}^{2}$ have a significant higher 1-year mortality [11]. Subgroup analysis in this study also suggested a higher risk of death in patients implanted with HVAD compared with patients implanted with HMII. This was not a randomized study of the two devices; hence confounding limits the ability to make conclusions. Nonetheless, a higher rate of cerebrovascular accident (CVA) and late RVF were observed with HVAD patients, which is consistent with previous studies $[12,13]$. It should be noted that most of the patients in the registry were implanted before the publication of papers demonstrating the importance of blood pressure control in HVAD patients to limit complications [14]. The adverse events profile of HMII appears more favorable since higher rates of GIB typically lead to higher probability to be transplanted. Consistently, patients who received a HTx in our study were more frequently supported with an HMII compared with an HVAD. Finally, temporary MCS before CF-LVAD implantation was a strong predictor of mortality with a HR of 1.58 , which is consistent with multiple other studies that demonstrate worse outcomes with INTERMACS 1 patients that undergo CF-LVAD $[15,16]$. All the variables identified in the current study as independently associated with early or late mortality in patients treated with CF-LVAD were also reported in the 2019 INTERMACS annual report (increasing recipient age, critical cardiogenic shock at time of CF-LVAD, renal dysfunction and higher $\mathrm{BMI}$ ), with the exception of the type of device. It should be noted that in the INTERMACS analysis included both centrifugal flow devices (HVAD and HM3) while our analysis only included HVAD.

\subsection{Study limitations}

The retrospective nature of our work is the primary limitation of our study. We also acknowledge potential bias in site selection with some heterogeneity in the number of cases provided by each participating center. While only 3 US centers were included in the registry, 1 -year survival was relatively similar between them and the most INTERMACS registry ( $83 \%$ vs. $87 \%$ respectively) [18], given we do not censure patients at the time of HTx. Similarly, the 1 year survival of European cohort is similar to the BTT cohort in EUROMACS (83\% vs. $78 \%$ respectively) [5]. Also, prevalence of comorbidities at baseline between the two cohorts was consistent with previous published data [19]. Thus although residual selection bias may still persist, the above data suggest a representative study population in the TRAViATA registry. Centers may also vary for specific features such as volume, training and experience of personnel, availability of resources and characteristics of center organization. Furthermore, as the patients at risk after 4 years of follow-up becomes less than 15\% of the initial cohort in the US group, statistical uncertainty of the esteem of survival is higher in the US cohort compared to EU group between 4 and 5 years of follow up [17].

We did not include the newest generation of CF-LVADs (HM3) as we wanted to include significant follow-up time in both cohorts. Further study will be required to validate our findings in HM3 patients. Furthermore, freedom from waitlist mortality or delisting at 1-year was $83.5 \%$ in patients mechanically supported in a UNOS analysis that considered
22,863 patients from 93 US HTx centers (period 2008-2015) [3]. Again, this figure was very close to the 1-year mortality reported in the 3 US centers in our study. Furthermore, another UNOS analysis on 5486 patients showed that patients with CF-LVAD at HTx spent a median of 191 days on the waitlist [11]. This figure is close to the time between CF-LVAD implant to HTx of 238 days observed in our US cohort.

In addition, some of laboratory tests which are related to RV failure (i.e. blood urea nitrogen) as well as medication regimens over time (i.e. anticoagulation and dressing protocol) that could explain differences in the adverse events rate in the two cohorts were not systematically obtained. Finally variables related to socio-economic status of patients, that could affect the outcome of LVAD patients were not collected in our registry. Similarly, we did not account for the impact on outcome of adverse events related or not with CF-LVAD that occurred in the follow up (for example occurrence of stroke or cancer).

In conclusion, in this large retrospective study of BTT CF-LVAD patients, outcomes are similar for US and EU patients. This is despite significant differences in the patient populations and organ availability. This suggests that MCS remains a a safe and effective tool for supporting patients waiting for HTx. Further prospective study is required to confirm these findings in more contemporary devices.

\section{Funding}

This work was partially supported by the US National Institutes of Health (NIH), Grant UL1TR001442 of CTSA funding. The content is solely the responsibility of the authors and does not necessarily represent the official views of the NIH.

\section{Declaration of Competing interest}

None.

\section{Acknowledgements}

The Authors thank Federico Landi for his contribution to the graphic design of Fig. 1.

\section{Appendix A. Supplementary data}

Supplementary Table 1 to 10 and Supplementary Fig. 1 to this article can be found online at https://doi.org/10.1016/j.ijcard.2020.09.026.

\section{References}

[1] JK Kirklin, FD Pagani, RL Kormos, LW Stevenson, ED Blume, SL Myers, et al., Eighth annual INTERMACS report: Special focus on framing the impact of adverse events, J. Heart Lung Transplant. 36 (10) (2017) 1080-1086 , Epub 2017/09/26 https:// doi.org/10.1016/j.healun.2017.07.00528942782.

[2] JK Kirklin, R Xie, J Cowger, T de By, T Nakatani, S Schueler, et al., Second annual report from the ISHLT Mechanically Assisted Circulatory Support Registry, J. Heart Lung Transplant. 37 (6) (2018) 685-691 , Epub 2018/03/20 https://doi.org/10. 1016/j.healun.2018.01.129429550146.

[3] L.K. Truby, A.R. Garan, R.C. Givens, K. Takeda, H. Takayama, P.N. Trinh, et al. Ventricular assist device utilization in heart transplant candidates: nationwide variability and impact on waitlist outcomes, Circ. Heart Fail. 11 (4) (2018) e004586 , Epub 2018/04/19 https://doi.org/10.1161/CIRCHEARTFAILURE.117.004586 PubMed PMID: 29666073; PubMed Central PMCID: PMCPMC5912344.

[4] L.W. Stevenson, Crisis awaiting heart transplantation: sinking the lifeboat, JAMA Intern. Med. 175 (8) (2015) 1406-1409 , Epub 2015/06/02 https://doi.org/10.1001/ jamainternmed.2015.2203 PubMed PMID: 26030521.

[5] T. de By, P. Mohacsi, B. Gahl, A. Zittermann, T. Krabatsch, F. Gustafsson, et al., The European registry for patients with mechanical circulatory support (EUROMACS) of the European association for cardio-thoracic surgery (EACTS): second report, Eur. J. Cardiothorac. Surg. 53 (2) (2018) 309-316, Epub 2017/10/14 https://doi. org/10.1093/ejcts/ezx320 PubMed PMID: 29029117.

[6] D.C. Reineke, P.J. Mohacsi, New role of ventricular assist devices as bridge to transplantation: European perspective, Curr. Opin. Organ Transplant 22 (3) (2017) 225-230, Epub 2017/04/01 https://doi.org/10.1097/MOT.0000000000000412 PubMed PMID: 28362668; PubMed Central PMCID: PMCPMC5427991. 
[7] E.J. Birks, The comparative use of ventricular assist devices: differences between Europe and the United States, Tex. Heart Inst. J. 37 (5) (2010) 565-567 Epub 2010/10/28. PubMed PMID: 20978570; PubMed Central PMCID: PMCPMC2953212.

[8] Y.K. Gernhofer, O.O. Braun, M. Brambatti, Q.M. Bui, J. Silva Enciso, B.H. Greenberg, et al., Which advanced heart failure therapy strategy is optimal for patients over 60 years old? J. Cardiovasc. Surg. 60 (2) (2019) 251-258, Epub 2018/09/01 10. 23736/S0021-9509.18.10593-3 PubMed PMID: 30168306.

[9] E.M. Hsich, L Thuita, D.M. McNamara, J.G. Rogers, M. Valapour LR Goldberg et al. Variables of importance in the Scientific Registry of Transplant Recipients database predictive of heart transplant waitlist mortality, Am. J. Transplant. (2019)https:// doi.org/10.1111/ajt.15265 , Epub 2019/01/20 30659754

[10] S.J. Forest, R. Xie, J.K. Kirklin, J. Cowger, Y. Xia, A.I. Dipchand, et al., Impact of body mass index on adverse events after implantation of left ventricular assist devices: an IMACS registry analysis, J. Heart Lung Transplant. 37 (10) (2018) 1207-1217, Epub 2018/09/10 https://doi.org/10.1016/j.healun.2018.06.004 PubMed PMID: 30195832; PubMed Central PMCID: PMCPMC6175660.

[11] L.K. Truby, M.A. Farr, A.R. Garan, R. Givens, S.W. Restaino, F. Latif, et al., Impact of bridge to transplant with continuous flow left ventricular assist devices on posttransplant mortality: a propensity matched analysis of the UNOS database, Circulation (2019)https://doi.org/10.1161/CIRCULATIONAHA.118.036932 Epub 2019/06/ 18. PubMed PMID: 31203669.

[12] JG Rogers, FD Pagani, AJ Tatooles, G Bhat, MS Slaughter, EJ Birks, et al., Intrapericardial Left Ventricular Assist Device for Advanced Heart Failure, The New England journal of medicine. 376 (5) (2017) 451-460 , Epub 2017/02/02 https://doi.org/10.1056/NEJMoa160295428146651.

[13] JM Stulak, ME Davis, N Haglund, S Dunlay, J Cowger, P Shah, et al., Adverse events in contemporary continuous-flow left ventricular assist devices: A multi-institutional comparison shows significant differences, The Journal of thoracic and cardiovascular surgery. 151 (1) (2016) 177-189, Epub 2015/11/08 https://doi.org/10.1016/j.jtcvs. 2015.09.10026545967.

[14] CA Milano, JG Rogers, AJ Tatooles, G Bhat, MS Slaughter, EJ Birks, et al., HVAD: The ENDURANCE Supplemental Trial, JACC Heart Fail. 6 (9) (2018) 792-802 , Epub 2018/07/17 https://doi.org/10.1016/j.jchf.2018.05.01230007559.

[15] RL Kormos, J Cowger, FD Pagani, JJ Teuteberg, DJ Goldstein, JP Jacobs, et al., The Society of Thoracic Surgeons Intermacs database annual report: Evolving indications, outcomes, and scientific partnerships, J. Heart Lung Transplant. 38 (2) (2019) 114-126, Epub 2019/01/30 https://doi.org/10.1016/j.healun.2018.11.01330691593.

[16] P Shah, FD Pagani, SS Desai, AJ Rongione, S Maltais, NA Haglund, et al., Outcomes of Patients Receiving Temporary Circulatory Support Before Durable Ventricular Assist Device, The Annals of thoracic surgery. 103 (1) (2017) 106-112 , Epub 2016/09/01 https://doi.org/10.1016/j.athoracsur.2016.06.00227577033.

[17] SJ Pocock, TC Clayton, DG Altman, Survival plots of time-to-event outcomes in clinical trials: good practice and pitfalls, Lancet. 359 (9318) (2002) 1686-1689 , Epub 2002/05/22 https://doi.org/10.1016/S0140-6736(02)08594-X12020548.

[18] J.K. Kirklin, D.C. Naftel, F.D. Pagani, R.L. Kormos, L.W. Stevenson, E.D. Blume, et al., Sixth INTERMACS annual report: a 10,000-patient database, J. Heart Lung Transplant. 33 (6) (2014) 555-564, https://doi.org/10.1016/j.healun.2014.04. 01024856259.

[19] P.C. Michaud, D. Goldman, D. Lakdawalla, A. Gailey, Y. Zheng Differences in health between Americans and Western Europeans: effects on longevity and public finance, Soc. Sci. Med. 73 (2) (2011) 254-263 , Epub 2011/07/02 https://doi.org/10. 1016/j.socscimed.2011.05.027 PubMed PMID: 21719178; PubMed Central PMCID: PMCPMC3383030. 